\title{
Homocysteine and long-term recurrent infarction following an acute coronary syndrome
}

\author{
Gema Miñana ${ }^{1,2 *}$, Carolina Gil-Cayuela ${ }^{2,3 *}$, Lorenzo Fácila ${ }^{4}$, Vicent Bodi ${ }^{1,2}$, \\ Ernesto Valero ${ }^{1,2}$, Anna Mollar¹, Maria Marco ${ }^{1}$, Teresa García-Ballester ${ }^{1}$, \\ Begoña Zorio ${ }^{1}$, Jorge Martí-Cervera ${ }^{5}$, Eduardo Núñez, \\ Francisco J. Chorro ${ }^{1,2}$, Juan Sanchis ${ }^{1,2}$, Julio Núñez ${ }^{1,2}$
}

${ }^{1}$ Cardiology Department, Hospital Clínico Universitario, Universitat de Valencia, INCLIVA, Valencia, Spain ${ }^{2}$ Centro de Investigación Biomédica en Red (CIBER-Cardiovascular),

Calle de Melchor Fernández Almagro, Madrid, Spain

${ }^{3}$ Cardiocirculatory Unit, Health Research Institute of La Fe University Hospital (IIS La Fe), Valencia, Spain ${ }^{4}$ Cardiology Departent, Consorcio Hospital General Universitario de Valencia, Valencia, Spain

${ }^{5}$ Universidad CEU Cardenal Herrera, Valencia, Spain

\section{Abstract}

Background: There are no well-established predictors of recurrent ischemic coronary events after an acute coronary syndrome (ACS). Higher levels of homocysteine have been reported to be associated with an increased atherosclerotic burden. The primary endpoint was to assess the relationship between homocysteine at discharge and very long-term recurrent myocardial infarction (MI).

Methods: 1306 consecutive patients with ACS were evaluated (862 with non-ST-segment elevation ACS [NSTEACS] and 444 with ST-segment elevation myocardial infarction [STEMI]) discharged from October 2000 to June 2003 in a single teaching-center. The relationship between homocysteine at discharge and recurrent MI was evaluated through bivariate negative binomial regression accounting for mortality as a competitive event.

Results: The mean age was $66.8 \pm 12.4$ years, $69.1 \%$ were men, and $32.2 \%$ showed prior diabetes mellitus. Most of the patients were admitted for an NSTEACS (66.0\%). The median (interquartile range) GRACE risk score, Charlson comorbidity index, and homocysteine were 144 (122-175) points, 1 (1-2) points, and $11.9(9.3-15.6) \mu \mathrm{mol} / \mathrm{L}$, respectively. In-hospital revascularization was performed in 26.3\% of patients. At a median follow-up of 9.7 (4.5-15.1) years, 709 (54.3\%) deaths were registered and 779 recurrent MI in 478 (36.6\%) patients. The rates of recurrent MI were higher in patients in the upper homocysteine quartiles $(p<0.001)$. After a multivariate adjustment, homocysteine along its continuum remained almost linearly associated with a higher risk of recurrent $M I(p=0.001)$ and all-cause mortality $(p<0.001)$.

Conclusions: In patients with ACS, higher homocysteine levels identified those at a higher risk of recurrent MI at very long-term follow-up. (Cardiol J 2021; 28, 4: 598-606)

Key words: homocysteine, acute coronary syndrome, recurrent myocardial infarction, coronary artery disease, risk factors

Address for correspondence: Julio Núñez, PhD, Cardiology Department, Hospital Clínico Universitario de Valencia, Avda. Blasco Ibáñez 17, 46010 Valencia, Spain, tel: +34 652856 689, e-mail: yulnunez@gmail.com

Received: 7.08.2020 Accepted: 6.10.2020

*Both authors contributed equally.

This article is available in open access under Creative Common Attribution-Non-Commercial-No Derivatives 4.0 International (CC BY-NC-ND 4.0) license, allowing to download articles and share them with others as long as they credit the authors and the publisher, but without permission to change them in any way or use them commercially. 


\section{Introduction}

Ischemic heart disease (IHD) remains the leading cause of death worldwide, despite advances in prevention, diagnosis, and treatment [1]. Identifying those at a higher risk of new cardiovascular ischemic events is essential for tailoring monitoring and therapeutics. Unfortunately, there are not well established biomarkers for predicting the risk of long-term recurrent myocardial infarction (MI) after an episode of acute coronary syndrome (ACS).

Mild elevations of homocysteine, a toxic sulfhydryl-containing amino acid formed during the demethylation of methionine, have been associated with an increased incidence of cardiovascular, cerebrovascular, or peripheral vascular diseases [2-5]. Although the exact biological pro-atherogenic effect of homocysteine remains to be determined, multiple mechanisms have been proposed. Among them, endothelial dysfunction, direct effects on platelets, smooth muscle proliferation, oxidative modification of low-density lipoproteins, endothelial-leukocyte interactions, and inhibition of fibrinolysis have been described in vitro and in vivo studies [6-9]. However, some studies failed to confirm the relationship between higher homocysteine and adverse clinical events $[10,11]$. Most of these studies were performed in a healthy population, with a median follow-up of up to 5 years, and evaluated time to a first event $[3,8,9]$. According to available research, there is no data in the literature endorsing the role of homocysteine for predicting long-term recurrent $\mathrm{MI}$ in patients with established IHD.

In this work, the aim was to evaluate whether homocysteine was associated with total long-term recurrent $\mathrm{MI}$ in a historical cohort of patients with ACS.

\section{Methods}

\section{Population and protocol}

A total of 1606 consecutive patients were hospitalized in a single-teaching center with a diagnosis of ACS from October 2000 to August 2003. None of these patients were transferred from other hospitals due to unsatisfactory clinical progress. Patients who died $(n=119)$ during the index admission were excluded from this analysis. Additionally, 181 patients without homocysteine assessment were excluded. Finally, 1306 patients comprised the final population for this analysis (862 with non-ST-segment elevation ACS [NSTEACS] and 444 with ST-segment elevation MI [STEMI]) (see flow chart in Suppl. Fig. 1). The baseline characteristics between those with and without homocysteine assessment are shown in Supplementary Table 1 . ACS definition and treatment were based on current guidelines operating at the time of patient inclusion [12-14]. The Charlson comorbidity index [15] and the Global Registry of Acute Coronary Events (GRACE) score [16] were determined in all patients.

For STEMI patients, fibrinolysis was the main reperfusion strategy at the time of enrolment. It was indicated in those presenting with ST-segment elevation (greater than $0.1 \mathrm{mV}$ in two or more contiguous leads) or new left bundle branch block and clinical history suggesting acute MI, and time to therapy of 12 hours or less. Rescue percutaneous coronary intervention was considered when the pharmacological reperfusion strategy failed [13, 14]. In patients with NSTEACS, an initial noninvasive strategy was applied. Cardiac catheterization during the index hospitalization was indicated in patients with persistent or recurrent episodes of symptomatic ischemia with or without associated electrocardiogram changes, and in those presenting shock, severe pulmonary congestion, or continuing hypotension $[13,14]$. The treatment strategy followed was established by current national and international guides operating at the time of the study $[13,14]$.

Written informed consent was obtained from each patient included in the study. The study protocol conforms to the ethical guidelines of the 1975 Declaration of Helsinki. The study protocol has been priorly approved by the Institution's ethics committee on research on humans.

\section{Blood samples}

Plasma total homocysteine at discharge, which includes the sum of protein-bound and free homocysteine, was measured by immunoassay of polarization of fluorescence (Axsym system, Abbott). The coefficients of variation within and between days for the analysis were $\leq 5 \%$.

\section{Outcome definition and follow-up}

Recurrent spontaneous MI was selected as the primary endpoint, whereas all-cause mortality was considered as a secondary endpoint. Patient clinical status and endpoint ascertainment were routinely evaluated by trained cardiologists during ambulatory clinic visits or through a review of the hospital or outpatient national electronic medical records. Only spontaneous MI was selected as an endpoint. Spontaneous MI was defined as an eleva- 
tion of myocardial markers (troponin I or creatine kinase-MB mass) associated with chest pain or compatible symptoms or ST-segment deviation [12]. Personnel in charge of events adjudication were blinded to the clinical data and exposures.

\section{Statistical analysis}

Continuous variables were expressed as mean \pm standard deviation (SD) or median (interquartile range [IQR]) when appropriate. Differences among homocysteine quartiles were tested with the ANOVA or Kruskal-Wallis rank test, respectively. Discrete variables were presented as percentages and compared with the $\chi^{2}$ test.

Time to death and first MI (adjusting for death as a competing event) across quartiles of homocysteine were plotted with the Kaplan-Meier and cumulative incidence function plots. Differences among the quartiles were tested with the log-rank test and Gray's test, respectively. The primary endpoint (recurrent MI) was evaluated by determining the incidence rate ratio (IRR), which is a risk estimate used for this type of method. To that end, a negative binomial regression was used to assess the association between homocysteine and the number of total recurrent MI during the entire follow-up. Because an increase of MI is most likely associated with an increased risk of subsequent death, it has been suggested that any analysis of recurrent admissions should also account for death as a competitive and terminal event. Thus, coefficients from this method were estimated by accounting for the positive correlation among the recurrent outcome and death as a terminal event, by linking the two simultaneous equations (rehospitalization count and death) with shared frailty [17]. Thus, within the same model, we obtain estimates of risk for both endpoints. Covariate selection was performed based on previous medical knowledge. The multivariable fractional polynomial method was used to determine the appropriate functional form of continuous covariates [18]. The covariates included in the final predictive model for both endpoints were: gender, type of ACS (NSTEACS vs. STEMI), GRACE risk score, Charlson comorbidity index, and revascularization during the index hospitalization.

A two-sided p-value of $<0.05$ was set as the threshold for statistical significance. All analyses were performed with Stata 15.1 (Stata Statistical Software, Release 15 [2017]; StataCorp LP, College Station, TX, USA).

\section{Results}

The mean age of the patients was $66.8 \pm 12.4$ years. Most of the patients were admitted for an NSTEACS (66.0\%) and showed troponin elevation (69.5\%). The proportion of males was $69.1 \%, 32.2 \%$ showed prior diabetes mellitus, $19.4 \%$ exhibited Killip class > I and $26.3 \%$ were revascularized during the index hospitalization. The median (IQR) GRACE risk score, Charlson comorbidity index, and homocysteine were 144 (122-175) points, 1 (1-2) points, and $11.9(9.3-15.6) \mu \mathrm{mol} / \mathrm{L}$, respectively.

\section{Baseline characteristics among homocysteine quartiles}

Baseline characteristics among homocysteine quartiles are presented in Table 1. Patients in the upper quartiles of homocysteine were older, more frequently males, and more often showed a history of hypertension, IHD, and Killip class > I during the index hospitalization. Also, they exhibited a higher GRACE score and the Charlson index.

\section{Homocysteine and risk of long-term recurrent MI}

At a median follow-up of 9.7 (4.5-15.1) years, $709(54.3 \%)$ deaths were registered and 779 recurrent MI in 478 (36.6\%) patients (Fig. 1). The number of recurrent MIs per patient were 1, 2, 3 and $>3$ in $299(22.9 \%), 112$ (8.6\%), 37 (2.8\%), and $32(2.3 \%)$ patients, respectively. Patients in the upper quartile of homocysteine showed the highest cumulative incidence rates of a first MI during the entire follow-up (Fig. 2). Similarly, the rates of total MI were also higher in patients in the upper quartile (per 100-person-year): 4.1, 3.6, 3.8, and 7.3 for Q1, Q2, Q3, and Q4, respectively $\mathrm{p}<0.001$ ). After a multivariate adjustment, including established prognosticators and accounting for death as a terminal event, homocysteine along its continuum remained almost linearly associated with a higher risk of recurrent MI $(\mathrm{p}=0.001)$, as is presented in Figure 3. When analyzed as quartiles, compared to those in the lower quartile, only patients in the upper quartile showed a significantly increased risk of recurrent MI (IRR $=1.42,95 \%$ confidence interval [CI] 1.11-1.81, $\mathrm{p}=0.005)$.

Subgroup analyses revealed a non-differential effect across most representative subgroups such as age ( $\leq 65$ vs. $>65$ years), gender, history of diabetes, prior IHD, type of ACS (NSTEACS vs. STEMI), and Charlson comorbidity index (above vs. below median), as depicted in Supplementary 
Table 1. Baseline characteristics among homocysteine quartiles.

\begin{tabular}{|c|c|c|c|c|c|}
\hline Variables & $\begin{array}{c}01 \\
(0.67-9.27 \\
\mu \mathrm{mol} / \mathrm{L}) \\
(\mathrm{n}=326)\end{array}$ & $\begin{array}{c}\mathrm{Q} 2 \\
(9.28-11.92 \\
\mu \mathrm{mol} / \mathrm{L}) \\
(\mathrm{n}=327)\end{array}$ & $\begin{array}{c}\text { Q3 } \\
(11.93-15.55 \\
\mu \mathrm{mol} / \mathrm{L}) \\
(\mathrm{n}=327)\end{array}$ & $\begin{array}{c}\text { Q4 } \\
(15.60-92.3 \\
\mu \mathrm{mol} / \mathrm{L}) \\
(\mathrm{n}=326)\end{array}$ & $\begin{array}{l}\text { P-value } \\
\text { for trend }\end{array}$ \\
\hline \multicolumn{6}{|l|}{ Demographics and medical history } \\
\hline Age [years] & $62.1 \pm 12.7$ & $65.5 \pm 11.0$ & $66.8 \pm 12.2$ & $73.0 \pm 11.0$ & $<0.001$ \\
\hline Sex (male) & $203(62.3 \%)$ & $230(70.3 \%)$ & $242(74.0 \%)$ & $228(69.9 \%)$ & 0.020 \\
\hline Hypertension & $176(54.0 \%)$ & $206(63.0 \%)$ & $197(60.2 \%)$ & $225(69.0 \%)$ & $<0.001$ \\
\hline Diabetes & $116(35.6 \%)$ & $106(32.4 \%)$ & $101(30.9 \%)$ & $97(29.7 \%)$ & 0.100 \\
\hline Dyslipidemia & $152(46.6 \%)$ & $139(42.5 \%)$ & $143(43.7 \%)$ & $122(37.4 \%)$ & 0.031 \\
\hline Smoker & $121(37.1 \%)$ & $104(31.8 \%)$ & $111(33.9 \%)$ & $72(20.1 \%)$ & $<0.001$ \\
\hline Prior smoker & $70(21.5 \%)$ & $85(26.0 \%)$ & $99(30.3 \%)$ & $101(31.0 \%)$ & 0.003 \\
\hline Family history of IHD & $45(13.8 \%)$ & $30(9.2 \%)$ & $24(7.3 \%)$ & $21(6.4 \%)$ & 0.001 \\
\hline Prior IHD & $105(32.2 \%)$ & $112(34.2 \%)$ & $124(37.9 \%)$ & $157(48.2 \%)$ & $<0.001$ \\
\hline Prior MI & $56(17.2 \%)$ & $69(21.1 \%)$ & $69(21.1 \%)$ & $91(27.9 \%)$ & 0.002 \\
\hline Previous $\mathrm{PCl}$ & $21(6.4 \%)$ & $18(5.5 \%)$ & $19(5.8 \%)$ & $14(4.3 \%)$ & 0.677 \\
\hline Previous CABG & $11(3.4 \%)$ & $18(5.5 \%)$ & $10(3.1 \%)$ & $18(5.5 \%)$ & 0.431 \\
\hline Charlson comorbidity index [points] ${ }^{\mathrm{a}}$ & $1(1-2)$ & $1(1-2)$ & $1(1-2)$ & $1.5(1-2)$ & $<0.001$ \\
\hline ACS type: & & & & & 0.210 \\
\hline $\begin{array}{l}\text { STEMI } \\
\text { NSTEACS }\end{array}$ & $\begin{array}{l}114(35.0 \%) \\
212(65.0 \%)\end{array}$ & $\begin{array}{l}116(35.5 \%) \\
211(64.5 \%)\end{array}$ & $\begin{array}{l}116(35.5 \%) \\
211(64.5 \%)\end{array}$ & $\begin{array}{c}98(30.1 \%) \\
228(69.9 \%)\end{array}$ & \\
\hline Killip class > I & $52(15.9 \%)$ & $46(14.1 \%)$ & $56(17.1 \%)$ & $99(30.4 \%)$ & $<0.001$ \\
\hline GRACE score [points] & $136(110-165)$ & $139(118-170)$ & $144(124-173)$ & $164(136-196)$ & $<0.001$ \\
\hline \multicolumn{6}{|l|}{ Vital signs on admission } \\
\hline Heart rate $[\mathrm{bpm}]$ & $85 \pm 19$ & $83 \pm 19$ & $84 \pm 17$ & $87 \pm 22$ & 0.073 \\
\hline $\mathrm{SBP}[\mathrm{mmHg}]$ & $145 \pm 26$ & $144 \pm 24$ & $147 \pm 24$ & $145 \pm 26$ & 0.542 \\
\hline \multicolumn{6}{|c|}{ Electrocardiogram and echocardiography } \\
\hline ST segment deviation & $175(53.7 \%)$ & $167(51.1 \%)$ & $173(52.9 \%)$ & $150(46.0 \%)$ & 0.088 \\
\hline LVEF [\% $]^{\mathrm{b}}$ & $60 \pm 13$ & $59 \pm 12$ & $58 \pm 13$ & $56 \pm 14$ & 0.002 \\
\hline \multicolumn{6}{|l|}{ Laboratory } \\
\hline Creatinine $[\mathrm{mg} / \mathrm{dL}]$ & $0.97 \pm 0.49$ & $1.05 \pm 0.40$ & $1.16 \pm 0.85$ & $1.62 \pm 1.23$ & $<0.001$ \\
\hline Total cholesterol $[\mathrm{mg} / \mathrm{dL}]^{\mathrm{c}}$ & $194 \pm 49$ & $194 \pm 38$ & $192 \pm 44$ & $192 \pm 41$ & 0.626 \\
\hline Troponin I elevation $(>1 \mathrm{ng} / \mathrm{mL})^{d}$ & $215(67.6 \%)$ & $216(67.5 \%)$ & $220(69.2 \%)$ & $236(75.5 \%)$ & 0.092 \\
\hline \multicolumn{6}{|c|}{ Revascularization during admission } \\
\hline Coronary angiography & $184(56.4 \%)$ & $162(49.5 \%)$ & $168(51.4 \%)$ & $121(37.1 \%)$ & $<0.001$ \\
\hline Revascularization & $105(32.2 \%)$ & $91(27.8 \%)$ & $87(26.6 \%)$ & $60(18.4 \%)$ & $<0.001$ \\
\hline
\end{tabular}

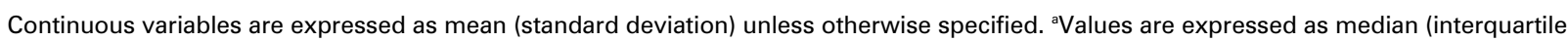
range); ${ }^{\mathrm{b}}$ Data available in $976(74.7 \%)$ patients; ${ }^{\circ}$ Data available in 1298 (99.4\%) patients; ${ }^{\mathrm{d}}$ Data available in 1277 (97.8\%) patients; ACS — acute coronary syndrome; CABG — coronary artery by-pass graft; GRACE — Global Registry of Acute Coronary Events; IHD - ischemic heart disease; LVEF — left ventricle ejection fraction; MI - myocardial infarction; NSTEACS - non-ST-segment elevation acute coronary syndrome; $\mathrm{PCI}$ - percutaneous coronary intervention; SBP - systolic blood pressure; STEMI - ST-segment elevation acute myocardial infarction

Figures 2, 3a and 3b. A significant differential association was found for the GRACE risk score (above vs. below median: $p$-value for interaction: 0.003). This interaction revealed the magnitude of the association between homocysteine and the risk of recurrent MI was greater in those with GRACE risk scores below the median (Suppl. Fig. 3c).
A sensitivity analysis, forcing treatments at discharge (antiplatelets [dual treatment with acetylsalicylic acid plus clopidogrel], renin-angiotensin-aldosterone inhibitors, beta-blockers, and statins) and hemoglobin (as a potential confounder) as covariates into the multivariate analysis, showed that homocysteine remained positively associated 


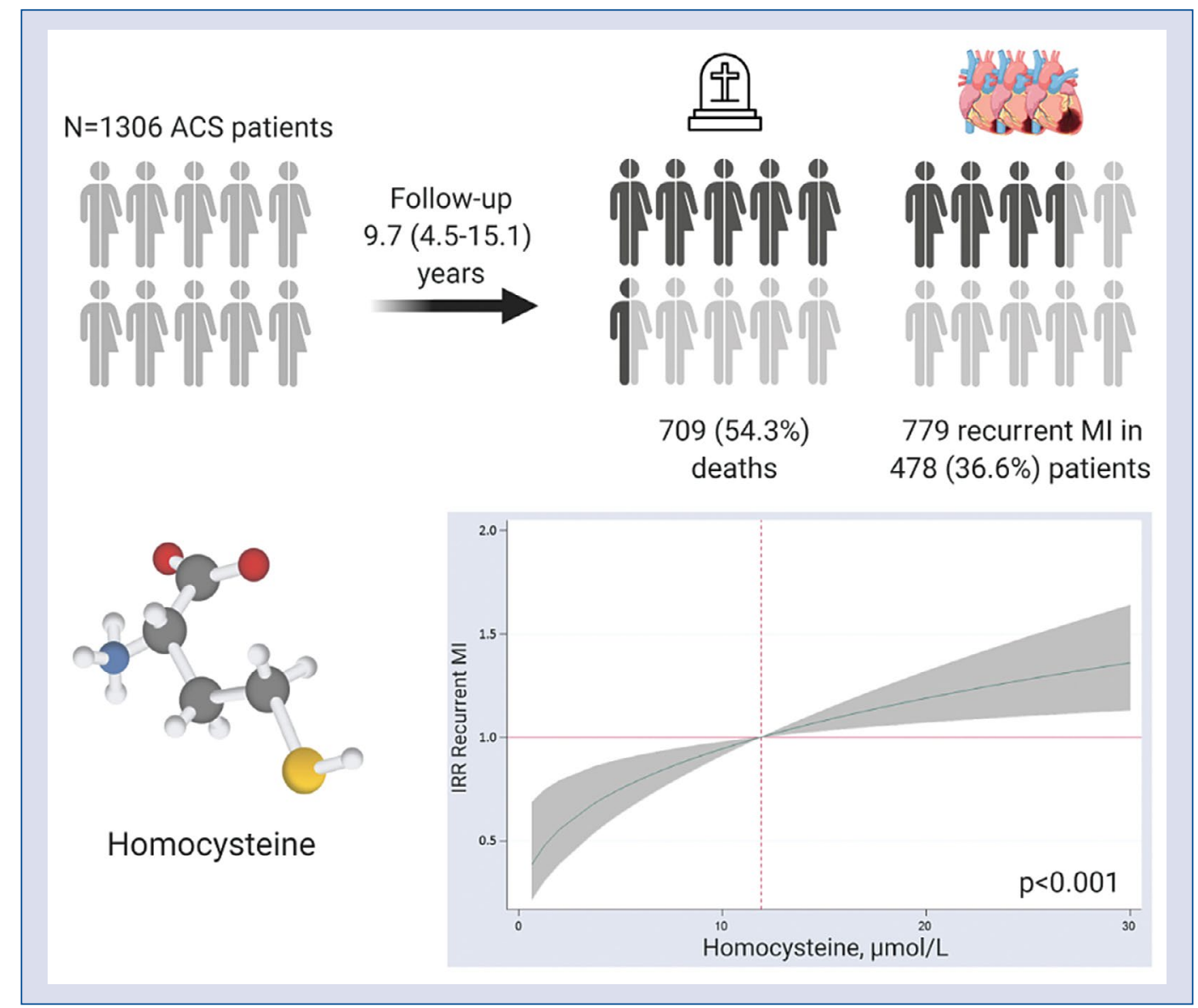

Figure 1. Graphical abstract.

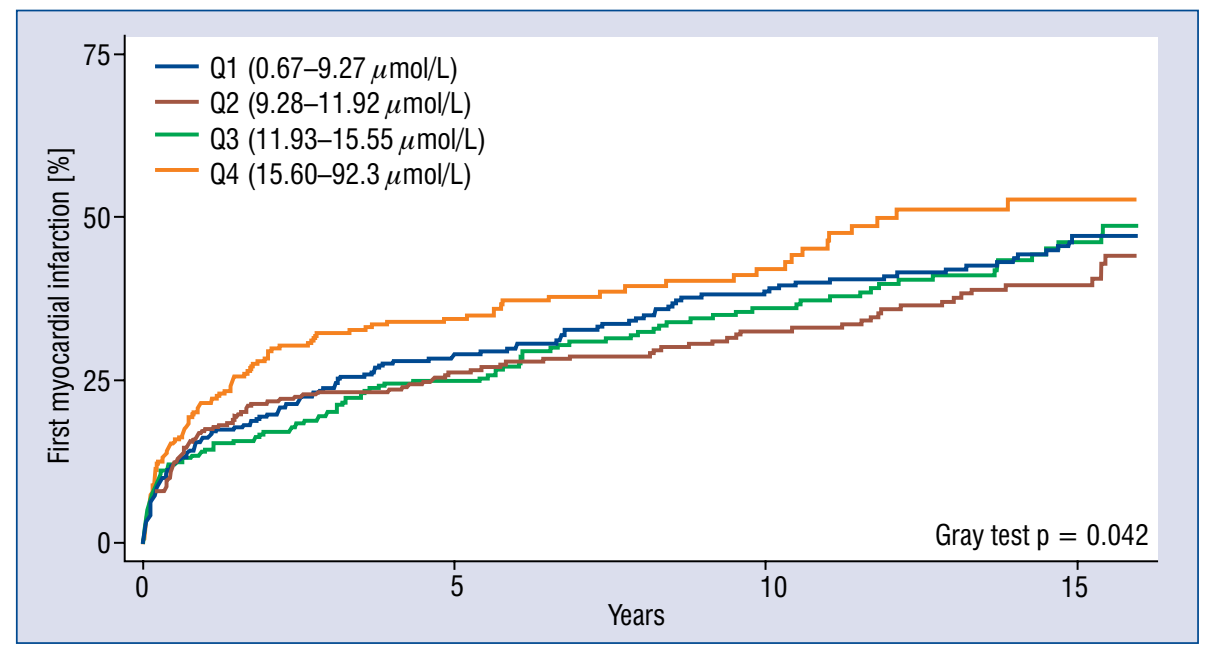

Figure 2. Time to a first myocardial infarction among homocysteine quartiles.

with the risk of MI $(p=0.002)$. Indeed, under this multivariate scenario, patients in the upper vs. lowest quartile displayed a significant excess of risk $(\mathrm{IRR}=1.41,95 \%$ CI 1.10-1.80, $\mathrm{p}=0.006)$. 


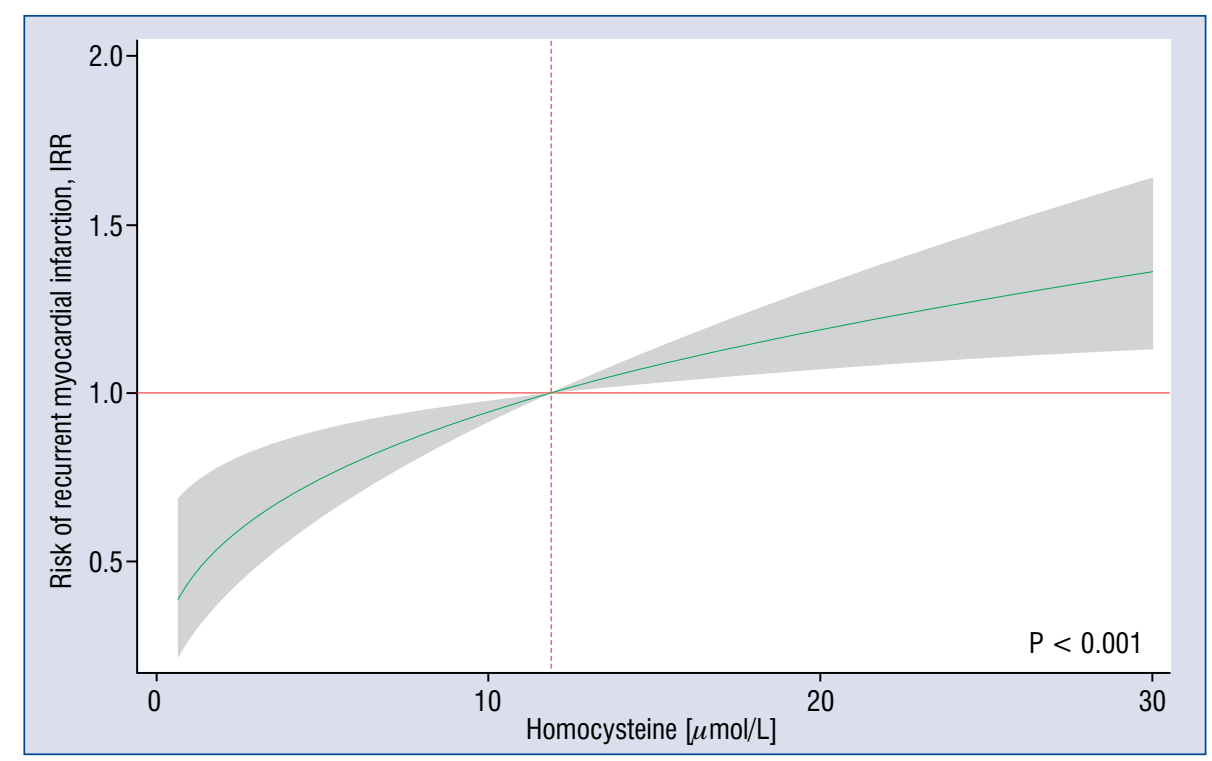

Figure 3. Homocysteine and baseline hazard of recurrent reinfarction; IRR — incidence rate ratio.

\section{Homocysteine and risk of long-term mortality}

During the follow-up, the incidence of death (per 100-person-year) significantly increased across homocysteine quartiles (3.7, 4.9, 5.7, and 10.7 for Q1, Q2, Q3, and Q4, respectively; $\mathrm{p}<0.001$ ). The Kaplan-Meier curves revealed a stepwise and sustained separation of the curves through the entire follow-up, especially for patients belonging to the upper quartile (Fig. 4A). Multivariate analysis confirmed that higher homocysteine during index admission was associated with a higher risk of death $(\mathrm{p}<0.001)$. This adjusted association also revealed an almost linear gradient of risk (Fig. 4B). When compared to patients in the lower quartile, adjusted-risk estimates showed a significative and stepwise increase of risk for Q2 (1.25, 95\% CI 0.95-1.63, $\mathrm{p}=0.107), \mathrm{Q} 3$ (1.41, 95\% CI $1.08-1.84, \mathrm{p}=0.012)$, and $\mathrm{Q} 4(2.00,95 \%$ CI 1.53-2.61, $\mathrm{p}<0.001)$.

\section{Discussion}

This work evaluated the relationship between homocysteine and recurrent MI and mortality at very long-term follow-up in a historical cohort of consecutive non-selected patients with an ACS. The main finding herein, is that those patients with higher homocysteine values, assessed during hospitalization for an ACS, showed a higher risk of recurrent $\mathrm{MI}$ and mortality. Both associations were independent of traditional and relevant prognosti- cators and potential confounders. These findings support the role of homocysteine in the pathogenesis of new acute coronary events in patients with coronary artery disease (CAD).

\section{Homocysteine and atherosclerosis}

Homocysteine, a sulfur-containing amino acid, is an intermediate product formed as a result of the catabolism of methionine. It is known that severe hyperhomocysteinemia ( $>100 \mu \mathrm{mol} / \mathrm{L}$ ) in patients with homozygous homocystinuria is associated with premature atherosclerosis [19]. Although this is a very rare pathology, minor elevated levels $(15-30 \mu \mathrm{mol} / \mathrm{L})$ have been described in up to $7 \%$ of the population [20].

The suggested mechanisms involved in homocysteine-induced atherosclerosis include: a) endothelial injury, which appears to be mediated by oxidative stress [21]; b) smooth muscle proliferation [22]; c) oxidative modification of low-density lipoproteins [6, 23, 24]; d) endothelial-leukocyte interactions [25]; e) reduced fibrinolytic activity $[9,26]$, and f) direct effects on platelets [27]. However, the specific weight of each of these mechanisms remains elusive, especially because many observations have been obtained from in vitro studies with homocysteine concentrations much higher than found in humans [8].

\section{Homocysteine and prognosis in CAD}

Several epidemiological studies have described a high prevalence of elevated plasma levels of homocysteine in patients with $\mathrm{CAD}$, stroke, 


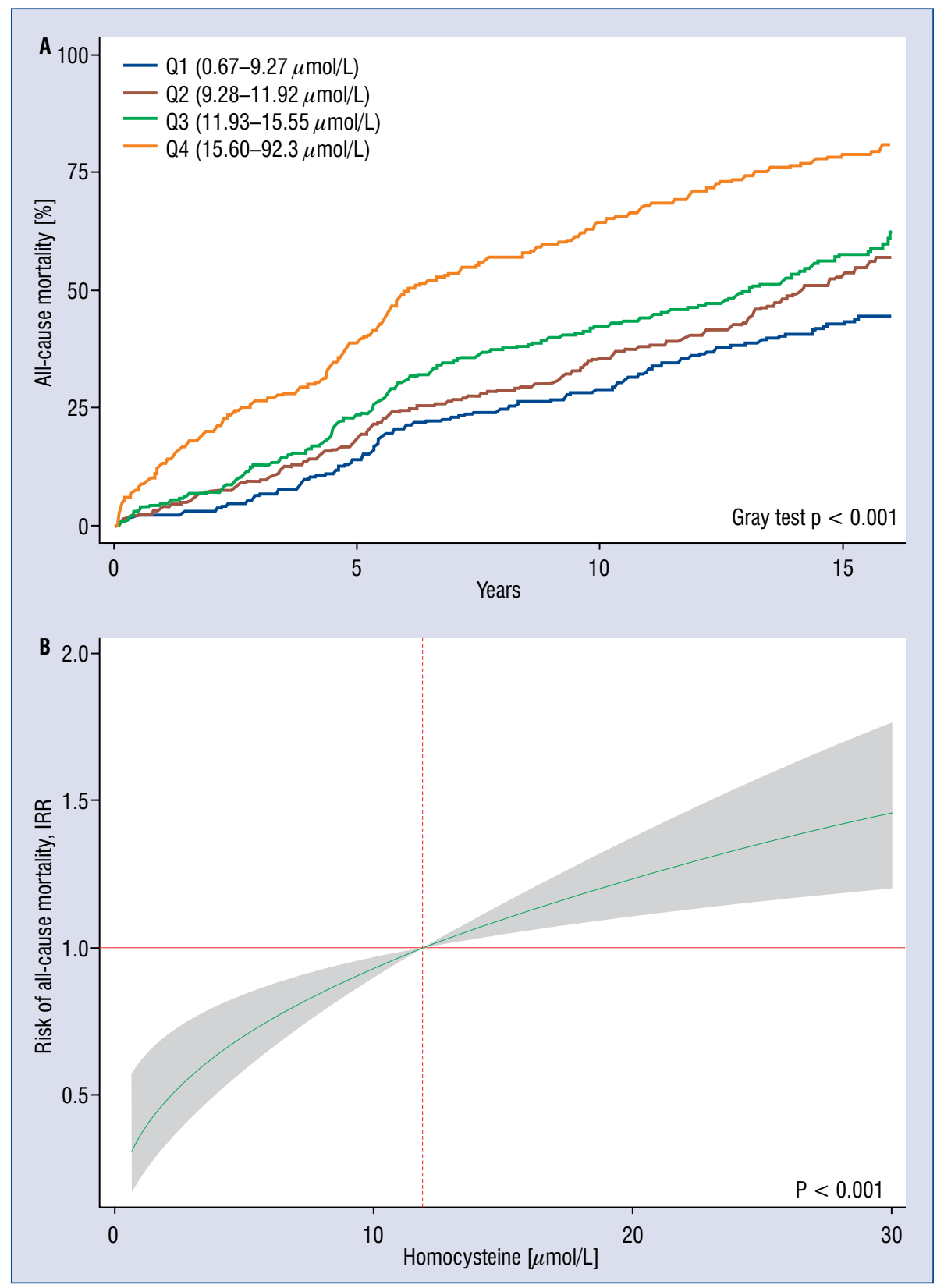

Figure 4. Homocysteine and all-cause mortality; A. Time to all-cause mortality among homocysteine quartiles; B. Baseline hazard of all-cause mortality; IRR — incidence rate ratio.

peripheral artery disease, and venous thrombosis [2-5]. Prospective studies correlated serum homocysteine levels to long-term outcomes in patients with STEMI [28], NSTACS [3], and without previous CAD [29]. In a meta-analysis, Boushey et al. [30] estimated that a $5-\mathrm{mmol} / \mathrm{L}$ homocysteine increment elevates $\mathrm{CAD}$ risk by as much as cholesterol increases of $0.5 \mathrm{mmol} / \mathrm{L}$. However, other authors failed to confirm this association [10,11]. For example, Ubbink et al. [11] showed no significant increase in $\mathrm{CAD}$ in those with higher homocysteine values in 2290 men in the Caerphilly cohort during a 5-year follow-up. Also, some randomized clinical trials, performed in subject with and without CAD, have failed to demonstrate any benefit in terms of reducing major cardiovascular events by lowering the homocysteine levels with diet supplementation with folic acid and B vitamins [31-33].

More recently, a higher risk of short-term mortality and nonfatal ischemic events has been reported in patients with NSTEACS [34] and STEMI [35]. In these studies, the risk of new recurrent coro- 
nary ischemic events at long-term follow-up was not addressed. Thus, this is the first study showing that higher levels of homocysteine are related to a higher risk of new coronary ischemic events.

\section{Clinical implications}

Reinfarction risk prediction in patients with established CAD is still an unmet need. The utility of homocysteine measurement after an index MI is not well-established. According to the present findings, higher levels of homocysteine may identify a subset of patients at a higher risk of new coronary ischemic events. These patients may probably benefit from a closer follow-up and more aggressive treatment. Further studies are necessary to confirm the present findings in cohorts with more contemporary treatments, elucidate the mechanisms behind these findings, and re-evaluate the long-term effects of therapies for reducing homocysteine in patients with prior ACS.

\section{Strengths and limitations}

The main strength of the present study is the very-long follow-up, which allowed recording a large number of repeated new ischemic coronary events. Some important limitations need to be addressed: a) this is an observational single-center study, which may influence the applicability of these results to other populations; b) the generalization of our findings to the current era is limited by the fact that revascularization strategies and medical treatment have substantially changed. In addition, given the low proportion of patients that received coronary artery bypass during the index admission, the potential differential effect of type of revascularization along the continuum of homocysteine could not be evaluated; c) we did not assess the revascularization procedures nor medical treatment changes during the post-discharge follow-up, which may operate as important confounders; d) finally, in this study the longitudinal trajectory of homocysteine was not measured, precluding to infer how its trajectory may be associated with the risk of recurrent MI. Nevertheless, it has been reported that the occurrence of an MI does not change homocysteine plasma levels [36].

\section{Conclusions}

In patients with ACS, higher homocysteine levels identified those at a higher risk of recurrent MI very long-term follow-up. The present study results provide new evidence about the utility of homocysteine as a potential risk predictor of new coronary ischemic events. Further contemporary studies are warranted to re-evaluate the applicability of the present findings to more contemporary cohorts and the role of homocysteine as a therapeutic target in this scenario.

\section{Funding}

This work was supported in part by grants from CIBER CV (grant number 16/11/00420) and Instituto de Salud Carlos III-FEDER (grant numbers PIE15/00013 and PI17/01836), Madrid, Spain. The authors have no other funding, financial relationships, or conflicts of interest to disclose relative to this work.

The funding sources were not involved in the study design, collection, analysis and interpretation of data in the writing of the report, and in the decision to submit the article for publication.

\section{Conflict of interest: None declared}

\section{References}

1. Benjamin EJ, Muntner P, Alonso A, et al. American Heart Association Council on E, Prevention Statistics C, Stroke Statistics S. Heart Disease and Stroke Statistics-2019 Update: A Report From the American Heart Association. Circulation. 2019; 139: e56-e528.

2. Selhub J. The many facets of hyperhomocysteinemia: studies from the Framingham cohorts. J Nutr. 2006; 136(6 Suppl): 1726S-1730S, doi: 10.1093/jn/136.6.1726S, indexed in Pubmed: 16702347.

3. Fácila L, Nuñez JE, G VB, et al. Early determination of homocysteine levels in acute coronary syndromes, is it an independent prognostic factor? Int J Cardiol. 2005; 100(2): 275-279, doi: 10.1016/j.ijcard.2004.09.001, indexed in Pubmed: 15823635.

4. Bostom AG, Rosenberg IH, Silbershatz H, et al. Nonfasting plasma total homocysteine levels and stroke incidence in elderly persons: the Framingham Study. Ann Intern Med. 1999; 131(5): 352-355, doi: 10.7326/0003-4819-131-5-199909070-00006, indexed in Pubmed: 10475888.

5. Anderson JL, Muhlestein JB, Horne BD, et al. Plasma homocysteine predicts mortality independently of traditional risk factors and C-reactive protein in patients with angiographically defined coronary artery disease. Circulation. 2000; 102(11): 1227-1232, doi: 10.1161/01.cir.102.11.1227, indexed in Pubmed: 10982535.

6. Holvoet P, Collen D. Oxidized lipoproteins in atherosclerosis and thrombosis. FASEB J. 1994; 8(15): 1279-1284, doi: 10.1096/ fasebj.8.15.8001740, indexed in Pubmed: 8001740.

7. McCully KS. Macromolecular basis for homocystein-induced changes in proteoglycan structure in growth and arteriosclerosis. Am J Pathol. 1972; 66(1): 83-96, indexed in Pubmed: 5009253.

8. ThambyrajahJ, TownendJN. Homocysteine and atherothrombosis-mechanisms for injury. Eur Heart J. 2000; 21(12): 967-974, doi: 10.1053/euhj.1999.1914, indexed in Pubmed: 10901508.

9. Tofler GH, D'Agostino RB, Jacques PF, et al. Association between increased homocysteine levels and impaired fibrinolytic potential: potential mechanism for cardiovascular risk. Thromb Haemost. 2002; 88(5): 799-804, indexed in Pubmed: 12428097. 
10. Folsom AR, Nieto FJ, McGovern PG, et al. Prospective study of coronary heart disease incidence in relation to fasting total homocysteine, related genetic polymorphisms, and B vitamins: the Atherosclerosis Risk in Communities (ARIC) study. Circulation. 1998; 98(3): 204-210, doi: 10.1161/01.cir.98.3.204, indexed in Pubmed: 9697819.

11. Ubbink JB, Fehily AM, Pickering J, et al. Homocysteine and ischaemic heart disease in the Caerphilly cohort. Atherosclerosis. 1998; 140(2): 349-356, doi: 10.1016/s0021-9150(98)00139-7, indexed in Pubmed: 9862278.

12. Myocardial infarction redefined: a consensus document of The Joint European Society of Cardiology/American College of Cardiology Committee for the redefinition of myocardial infarction. Eur Heart J. 2000; 21(18): 1502-1513, doi: 10.1053/ euhj.2000.2305, indexed in Pubmed: 10973764.

13. Ryan TJ, Antman EM, Brooks NH, et al. 1999 update: ACC/AHA guidelines for the management of patients with acute myocardial infarction. A report of the American College of Cardiology/ /American Heart Association Task Force on Practice Guidelines (Committee on Management of Acute Myocardial Infarction). J Am Coll Cardiol. 1999; 34: 890-911.

14. Ryan T, Anderson J, Antman E, et al. ACC/AHA guidelines for the management of patients with acute myocardial infarction. J Am Coll Cardiol. 1996; 28(5): 1328-1419, doi: 10.1016/s07351097(96)00392-0.

15. Tang EW, Wong CK, Herbison P. Global Registry of Acute Coronary Events (GRACE) hospital discharge risk score accurately predicts long-term mortality post acute coronary syndrome. Am Heart J. 2007; 153(1): 29-35, doi: 10.1016/j.ahj.2006.10.004, indexed in Pubmed: 17174633.

16. Charlson ME, Pompei P, Ales KL, et al. A new method of classifying prognostic comorbidity in longitudinal studies: development and validation. J Chronic Dis. 1987; 40(5): 373-383, doi: 10.1016/0021-9681(87)90171-8, indexed in Pubmed: 3558716.

17. Xu X, Hardin J. Regression models for bivariate count outcomes. Stata J. 2018; 16(2): 301-315, doi: 10.1177/1536867x1601600203.

18. Royston P, Sauerbrei W. Multivariable Model-Building: A Pragmatic Approach to Regression Analysis Based on Fractional Polynomials for Modelling Continuous Variables. Wiley, Chichester, UK 2008 .

19. McCully KS. Vascular pathology of homocysteinemia: implications for the pathogenesis of arteriosclerosis. Am J Pathol. 1969; 56(1): 111-128, indexed in Pubmed: 5792556.

20. Ueland PM, Refsum H. Plasma homocysteine, a risk factor for vascular disease: plasma levels in health, disease, and drug therapy. J Lab Clin Med. 1989; 114(5): 473-501, indexed in Pubmed: 2681479 .

21. Berman RS, Martin W. Arterial endothelial barrier dysfunction: actions of homocysteine and the hypoxanthine-xanthine oxidase free radical generating system. Br J Pharmacol. 1993; 108(4): 920-926, doi: 10.1111/j.1476-5381.1993.tb13487.x, indexed in Pubmed: 8485631.

22. Heinecke JW, Rosen H, Suzuki LA, et al. The role of sulfurcontaining amino acids in superoxide production and modification of low density lipoprotein by arterial smooth muscle cells. J Biol Chem. 1987; 262(21): 10098-10103, indexed in Pubmed: 3038867.

23. Naruszewicz M, Mirkiewicz E, Kłosiewicz-Latoszek L. Modification of low density lipoproteins from hypertriglyceridemic patients by macrophages in vitro and the effect of bezafibrate treatment. Atherosclerosis. 1989; 79(2-3): 261-265, doi: 10.1016/0021-9150(89)90133-0, indexed in Pubmed: 2597235 .
24. Parthasarathy S. Oxidation of low-density lipoprotein by thiol compounds leads to its recognition by the acetyl LDL receptor. Biochim Biophys Acta. 1987; 917(2): 337-340, doi: 10.1016/00052760(87)90139-1, indexed in Pubmed: 3801507.

25. Dudman NP, Temple SE, Guo XW, et al. Homocysteine enhances neutrophil-endothelial interactions in both cultured human cells and rats In vivo. Circ Res. 1999; 84(4): 409-416, doi: 10.1161/01. res.84.4.409, indexed in Pubmed: 10066675.

26. Speidl WS, Nikfardjam M, Niessner A, et al. Mild hyperhomocysteinemia is associated with a decreased fibrinolytic activity in patients after ST-elevation myocardial infarction. Thromb Res. 2007; 119(3): 331-336, doi: 10.1016/j.thromres.2006.02.011, indexed in Pubmed: 16616324.

27. McDonald L, Bray C, Field C, et al. Homocystinuria, thrombosis, and the blood-platelets. Lancet. 1964; 1(7336): 745-746, doi 10.1016/s0140-6736(64)92852-1, indexed in Pubmed: 14107984.

28. Fan Y, Wang J, Zhang S, et al. Homocysteine enhances the predictive value of the GRACE risk score in patients with ST-elevation myocardial infarction. Anatol J Cardiol. 2017; 18(3): 182-193, doi: 10.14744/AnatolJCardiol.2017.7798, indexed in Pubmed: 28782750 .

29. Acevedo M, Pearce GL, Jacobsen DW, et al. Serum homocysteine levels and mortality in outpatients with or without coronary artery disease: an observational study. Am J Med. 2003; 114(8): 685-688, doi: 10.1016/s0002-9343(03)00123-2, indexed in Pubmed: 12798457.

30. Boushey CJ, Beresford SA, Omenn GS, et al. A quantitative assessment of plasma homocysteine as a risk factor for vascular disease. Probable benefits of increasing folic acid intakes. JAMA. 1995; 274(13): 1049-1057, doi: 10.1001/ jama.1995.03530130055028, indexed in Pubmed: 7563456.

31. Study of the Effectiveness of Additional Reductions in C, Homocysteine Collaborative G, Armitage JM, Bowman L, Clarke RJ, Effects of homocysteine-lowering with folic acid plus vitamin B12 vs placebo on mortality and major morbidity in myocardial infarction survivors: a randomized trial. JAMA. 2010; 303: 2486-2494, doi: 10.1001/jama.2010.840, indexed in Pubmed: 20571015.

32. Albert CM, Cook NR, Gaziano JM, et al. Effect of folic acid and $B$ vitamins on risk of cardiovascular events and total mortality among women at high risk for cardiovascular disease: a randomized trial. JAMA. 2008; 299(17): 2027-2036, doi: 10.1001/ jama.299.17.2027, indexed in Pubmed: 18460663.

33. Toole JF, Malinow MR, Chambless LE, et al. Lowering homocysteine in patients with ischemic stroke to prevent recurrent stroke, myocardial infarction, and death: the Vitamin Intervention for Stroke Prevention (VISP) randomized controlled trial. JAMA. 2004; 291(5): 565-575, doi: 10.1001/jama.291.5.565, indexed in Pubmed: 14762035.

34. Hultdin J, Thøgersen AM, Jansson JH, et al. Elevated plasma homocysteine: cause or consequence of myocardial infarction? J Intern Med. 2004; 256(6): 491-498, doi: 10.1111/j.13652796.2004.01415.x, indexed in Pubmed: 15554950.

35. Nevado JB, Imasa MS. Homocysteine predicts adverse clinical outcomes in unstable angina and non-ST elevation myocardial infarction: implications from the folate intervention in non-ST elevation myocardial infarction and unstable angina study. Coron Artery Dis. 2008; 19(3): 153-161, doi: 10.1097/ MCA.0b013e3282f52910, indexed in Pubmed: 18418231.

36. Ma Yi, Li Li, Geng XB, et al. Correlation between hyperhomocysteinemia and outcomes of patients with acute myocardial infarction. Am J Ther. 2016; 23(6): e1464-e1468, doi: 10.1097/ MJT.0000000000000130, indexed in Pubmed: 25405897. 